\title{
Absolute Change in High-Sensitivity Cardiac Troponin I at Three Hours After Presentation is Useful for Diagnosing Acute Myocardial Infarction in the Emergency Department
}

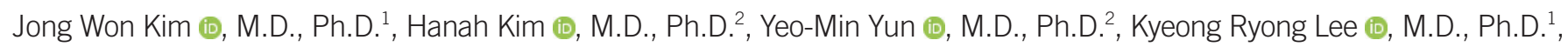
and Hyun Joong Kim $\mathbb{E}$, M.D., Ph.D. ${ }^{3}$

Departments of ${ }^{1}$ Emergency Medicine, ${ }^{2}$ Laboratory Medicine, ${ }^{3}$ Division of Cardiology, Department of Internal Medicine, Konkuk University School of Medicine, Konkuk University Medical Center, Seoul, Korea

Background: A rise and/or fall in cardiac troponin value with at least one value above the 99th percentile upper reference limit is essential for acute myocardial infarction (AMI) diagnosis. We evaluated the clinical usefulness of serial high-sensitivity cardiac troponin I (hs-cTnl) measurements in AMI diagnosis, in terms of the predictability of absolute and relative changes.

Methods: For this retrospective, forward observational study, we enrolled 281 patients older than 18 years who presented with chest pain at the emergency department (ED) between August 2015 and December 2016. The patients were grouped as AMI and nonAMI, and 73 (26\%) were diagnosed as having AMI. Hs-cTnl (Abbott Diagnostics, Abbott Park, IL, USA) was measured at presentation and 3 hours later. We assessed the diagnostic performance of the absolute and relative changes in hs-cTnl.

Results: The cut-off values to predict AMI were $16.2 \mathrm{ng} / \mathrm{L}$ and $42.1 \%$ for the absolute and relative hs-cTnl changes, respectively. The area under the curve of hs-cTnl for AMI diagnosis was larger for absolute changes than for relative changes [0.96 (95\% confidence interval [Cl], 0.92-0.98) vs 0.89 (95\% Cl, 0.85-0.93)] ( $P=0.014)$.

Conclusions: The absolute hs-cTnl change at 3 hours after presentation was superior to the relative change, and a rise and/or fall in hs-cTnl of $>16.2 \mathrm{ng} / \mathrm{L}$ at 3 hours after presentation was useful to identify AMI in patients presenting at the ED.

Key Words: Acute myocardial infarction, Chest pain, High-sensitivity cardiac troponin I, Emergency department, Absolute change
Received: January 22, 2020

Revision received: March 30, 2020

Accepted: June 1, 2020

\section{Corresponding author:}

Hanah Kim, M.D., Ph.D.

Department of Laboratory Medicine,

Konkuk University Medical Center, 120-1

Neungdong-ro, Gwangjin-gu, Seoul 05030, Korea

Tel: +82-2-2030-7826

Fax: +82-2-2030-5590

E-mail: md.hkim@gmail.com

\section{INTRODUCTION}

Acute coronary syndromes (ACS) are life-threatening disorders associated with high mortality and morbidity, despite advances in diagnosis and treatment [1]. Many patients with ACS present at the emergency department (ED) with chest pain [2]. Early and accurate identification of acute myocardial infarction (AMI) in these patients is crucial for providing proper treatment and improving prognosis [3]. The Fourth Universal Definition of Myocardial Infarction (2018) defines AMI as the presence of acute myocardial injury, detected by abnormal cardiac biomarkers, with clinical evidence of acute myocardial ischemia [4]. Especially, cardiac troponin (cTn) is preferred over creatine kinase, creatine kinase myocardial band isoform, or myoglobin as bio- 
markers for the evaluation of myocardial injury, which is considered when an elevation of cTn above the 99th percentile upper reference limit (URL) is detected [4]. The detection of a rise or a fall in the cTn value is required to distinguish acute myocardial injury from a chronic one [5-7]. Non-ischemic myocardial injury may arise secondary to various cardiac conditions, such as myocarditis, or may be associated with noncardiac conditions, such as renal failure, cardiorenal syndrome, and metabolic syndrome [8-10]. In addition, the clinicians could discriminate acute injury from chronic cTn elevation condition based on a rise and/or fall in the cTn values. Therefore, serial measurements of cTn value using high-sensitivity cTn (hs-cTn) assays are incorporated in the guidelines of the European Society of Cardiology (ESC) as well as the Fourth Universal Definition of Myocardial Infarction (2018) [4, 11, 12]. Both absolute and relative changes in hscTn value between presentation in ED and 3 hours later are used to confirm acute events and improve the specificity of hscTn assays; however, it is unclear whether the absolute or relative change in hs-cTn is more appropriate to distinguish true myocardial injury [4, 13-19].

In this study, we aimed to investigate the clinical utility of absolute and relative changes in hs-cTnl at 3 hours after presenta- tion in the ED for the diagnosis of AMI in patients suspected of having non-ST-elevation ACS in a Korean population. Further, we established the cut-off value of the absolute or relative change for AMI diagnosis.

\section{MATERIALS AND METHODS}

\section{Study design and study population}

We performed this retrospective forward observational study in the ED of the Konkuk University Medical Center, Seoul, Korea from August 2015 to December 2016. We consecutively recruited patients older than 18 years presenting symptoms suggestive of ACS, such as chest pain, chest discomfort, and epigastric discomfort, without a final diagnosis. In total, 440 patients were enrolled in this study. We excluded 81 patients who showed an onset of symptoms longer than 12 hours ago and 78 patients presenting with ST-segment elevation on 12-lead electrocardiogram (ECG). Of the remaining 281 patients, 73 (26\%) patients were diagnosed as having AMI, and 208 (74\%) patients were classified as non-AMI. The non-AMI group included 37 (18\%) patients with unstable angina, 65 (31\%) patients with cardiac symptoms from causes other than coronary artery disease, and

Table 1. Baseline characteristics of patients

\begin{tabular}{|c|c|c|c|c|}
\hline & \multirow{2}{*}{ AMI $(N=73)$} & \multicolumn{2}{|c|}{ Non-AMI (N=208) } & \multirow{2}{*}{$P$} \\
\hline & & $\mathrm{UA}(\mathrm{N}=37)$ & Non-ACS (N=171) & \\
\hline Male & $58(79.5)$ & $21(56.8)$ & $107(62.6)$ & 0.006 \\
\hline Age (yr) & $63(54-77)$ & $67(59-76)$ & $55(46-69)$ & $<0.001$ \\
\hline \multicolumn{5}{|l|}{ Risk factor } \\
\hline Hypertension & $41(56.2)$ & $20(54.1)$ & $67(39.2)$ & 0.02 \\
\hline Diabetes & $23(31.5)$ & $14(37.8)$ & $35(20.5)$ & 0.03 \\
\hline Hyperlipidemia & $18(24.7)$ & $16(43.2)$ & $33(19.3)$ & 0.008 \\
\hline Past history of AMI & $13(17.8)$ & $13(35.1)$ & $14(8.2)$ & $<0.001$ \\
\hline $\mathrm{eGFR}^{*}\left(\mathrm{~mL} / \mathrm{min} / 1.73 \mathrm{~m}^{2}\right)$ & $76(60-87)$ & $77(59-87)$ & $83.5(64-90)$ & 0.014 \\
\hline Time of symptom onset ( $<3 \mathrm{hr}$ ) & $29(39.7)$ & $13(35.1)$ & $59(34.5)$ & 0.7 \\
\hline Time to 2nd sample (min) & $139(108-180)$ & $130(105-214)$ & $154(106-181)$ & 0.8 \\
\hline \multicolumn{5}{|l|}{ ECG at presentation } \\
\hline ST change & $36(49.3)$ & $15(40.5)$ & $48(28)$ & 0.005 \\
\hline LBBB & $3(4.1)$ & $1(2.7)$ & $1(0.6)$ & 0.1 \\
\hline RBBB & $5(6.8)$ & $1(2.7)$ & $12(7.0)$ & 0.6 \\
\hline № significant change & $29(39.7)$ & $20(54.1)$ & $110(64.3)$ & 0.002 \\
\hline
\end{tabular}

Data are presented as the median (interquartile range) or number (percentage).

$P$ was derived using the chi-squared test or Fisher's exact test to compare AMI, UA, and non-ACS patients.

*eGFR was calculated using the Modification of Diet in Renal Disease Study equation.

Abbreviations: AMI, acute myocardial infarction; eGFR, estimated glomerular filtration rate; LBBB, left bundle branch block; Non-ACS, non-acute coronary syndrome; RBBB, right bundle branch block; UA, unstable angina; ECG, electrocardiogram. 
106 (51\%) patients with non-cardiac symptoms or atypical symptoms. Patient characteristics are presented in Table 1.

We assessed the clinical information of all patients, including their medical history, physical examination, 12-lead ECG, chest radiogram, and routine blood tests at presentation and 3 hours later. For data analysis, two cardiologists independently reviewed all available above-mentioned clinical data. While blinded to the 2nd hs-cTnl value, the two cardiologists determined the final diagnosis as follows: detection of a rise and/or fall in hs-cTnl values, with at least one value above the 99th percentile URL and with at least one of the followings: symptoms of myocardial ischemia, new ischemic ECG changes, development of pathological $Q$ waves, imaging evidence of new regional wall motion abnormality in a pattern consistent with an ischemic etiology, or identification of a coronary thrombus by angiography [4]. All patients were categorized as AMI or non-AMI. AMI was defined according to The Fourth Universal Definition of Myocardial Infarction (2018) as the presence of evidence of myocardial necrosis in a clinical situation related to myocardial ischemia; all other patients were classified as non-AMI [4]. The non-AMI group included patients with unstable angina and non-ACS. The attending cardiologists decided on the treatment of patients according to the ESC guidelines [13].

There was no study-specific intervention or additional blood collection in this study. The study protocol was designed following the criteria of the Declaration of Helsinki. The Institutional Review Board of Konkuk University Medical Center, Seoul, Korea, exempted approval of the study protocol and waived the requirement to obtain informed consent from the patients enrolled in the study.

\section{hs-cTnl assay}

Blood was collected from the patient in a VACUETTE ${ }^{\circledR}$ LH lithium heparin separator tube (Greiner Bio-One GmbH, Kremsmünster, Austria) at the time of presentation at the $E D$ and 3 hours later. The hs-cTnl value was measured in fresh blood using the ARCHITECT STAT High Sensitive Troponin-I chemiluminescence immunoassay on the ARCHITECT i2000SR analyzer (Abbott Diagnostics, Abbott Park, IL, USA). According to a previous study, the limit of blank of the assay was $0.5 \mathrm{ng} / \mathrm{L}$, and the limit of detection was $1.4 \mathrm{ng} / \mathrm{L}$. The 99th percentile (CV\%) medical decision point was $20.7 \mathrm{ng} / \mathrm{L}$ in males and $16.1 \mathrm{ng} / \mathrm{L}$ in females; assay CVs at the 99th percentile ranged from 5.5\% to $6.4 \%$ [20]. During the study period, three levels of quality-control materials provided by the manufacturer were run daily, and the mean within-laboratory imprecision was $<4.2 \%$. The linear- ity range of hs-cTnl measurement was $0-49.4 \mathrm{ng} / \mathrm{L}$, and the carry-over was $\leq 1.0 \%$.

\section{Statistical analysis}

Data are presented as the median with interquartile range for continuous variables and as number (percentage) for categorical variables. The AMI group and the non-AMI group, which included patients with unstable angina and non-ACS, were compared using the Kruskal-Wallis test with post-hoc tests, the chisquare test and Fisher's exact test. Receiver operating characteristic (ROC) curves were generated to compare the AMI diagnostic performance of the absolute and relative changes in hscTnl at presentation and after 3 hours, and the area under the ROC curve $(A \cup C)$ values were computed. The sensitivity and specificity were computed based on the optimal cut-off values derived from the ROC curves. The data were analyzed using IBM SPSS Statistics for Windows, version 19.0 (IBM Corp., Armonk, NY) and MedCalc Statistical Software version 19.2 (MedCalc Software bvba, Ostend, Belgium). All statistical tests were two-sided, and $P<0.05$ was considered significant.

\section{RESULTS}

In the AMI group, hs-cTnl values differed significantly at presentation and 3 hours later (94.0 vs $611.4 \mathrm{ng} / \mathrm{L}, P<0.001$ ). In the non-AMI group, the values at the two time points were not significantly different (2.6 vs $4.1 \mathrm{ng} / \mathrm{L}, P=0.09$ ) (Fig. 1).

The absolute change in hs-cTnl at 3 hours after presentation was significantly higher in the AMI group than in the non-AMI group (272.6 vs $0.8 \mathrm{ng} / \mathrm{L}, P<0.001$; data not shown). The relative change in hs-cTnl at 3 hours after presentation was significantly higher in the AMI group than in the non-AMI group (436.9\% vs $9.9 \%, P<0.001$; data not shown).

The AMI diagnostic performance based on the AUC value was 0.96 [95\% confidence interval $(\mathrm{Cl}), 0.92-0.98]$ for the absolute change and $0.89(95 \% \mathrm{Cl}, 0.85-0.93)$ for the relative change. Absolute change showed significant differences relative to the other values (vs hs-cTnl at presentation, $P<0.001$; vs hs$c T n l$ at 3 hours after presentation, $P<0.001$; vs relative change, $P=0.003$ ). The optimal cut-off value of the absolute change at 3 hours after presentation was $16.2 \mathrm{ng} / \mathrm{L}$, and the sensitivity and specificity were $98.6 \%$ and $84.7 \%$, respectively. The optimal cut-off value of the relative change at 3 hours after presentation was $42.1 \%$, and the sensitivity and specificity were $81.9 \%$ and $80.9 \%$, respectively (Fig. 2). 

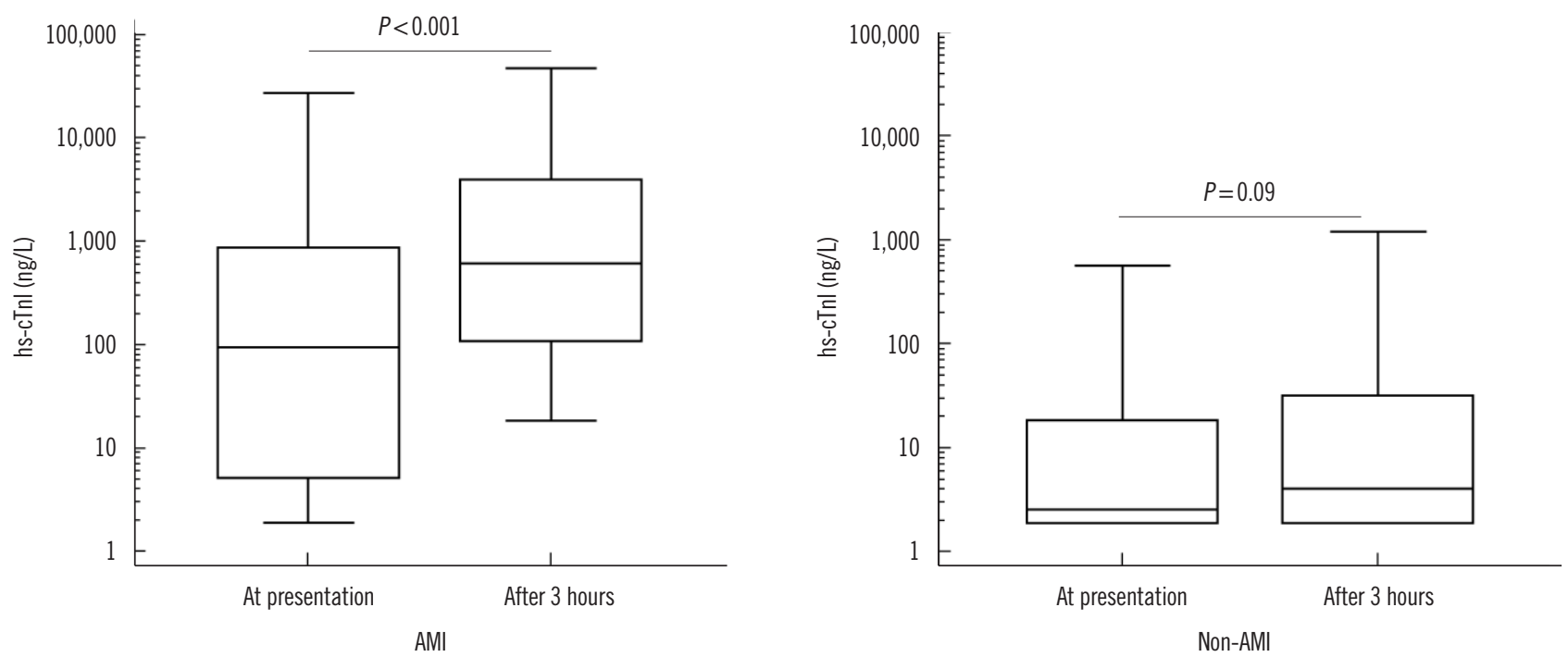

Fig. 1. Distribution of hs-cTnl values at presentation and 3 hours later in AMI and non-AMI groups. In each graph, the Y-axis is presented as a logarithmic scale. The central box represents the values from the lower to upper quartile (25th to 75th percentile). The middle line represents the median value. The vertical line extends from minimum to maximum values.

Abbreviations: AMI, acute myocardial infarction; hs-cTnl, high-sensitivity cardiac troponin I.

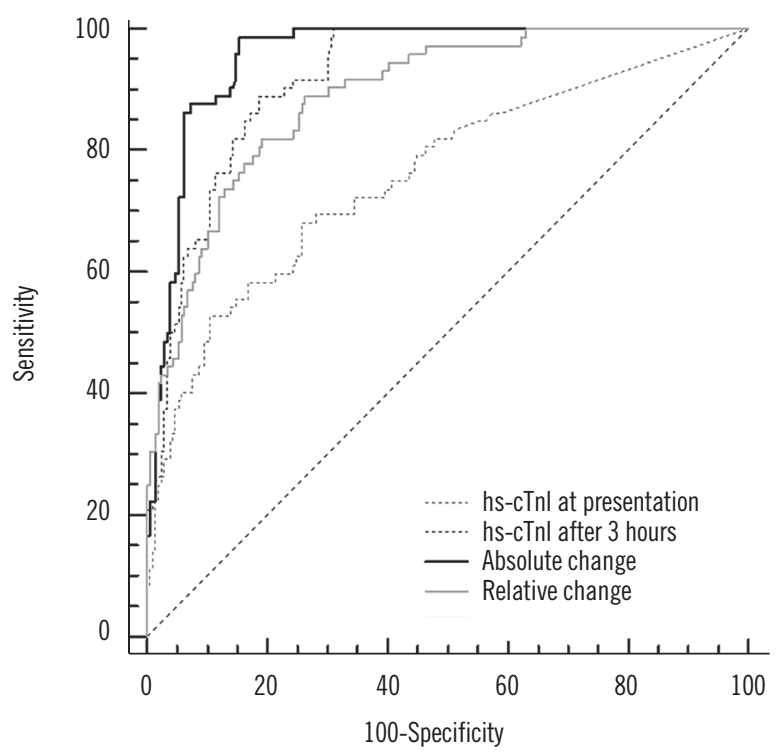

Fig. 2. ROC curves for the hs-cTnl value at presentation, the hscTnl value at 3 hours after presentation, the absolute change in hs-cTnl, value and the relative change in hs-cTnl value for the diagnosis of AMI. Absolute change showed significant differences relative to the other values (vs hs-cTnl at presentation, $P<0.001$; vs hs-cTnl at 3 hours after presentation, $P<0.001$; vs relative change, $P=0.003$ ).

Abbreviations: AMI, acute myocardial infarction; AUC, area under the curve; $\mathrm{Cl}$, confidence interval; hs-cTnl, high-sensitivity cardiac troponin I; ROC, receiver-operating characteristic.

\begin{tabular}{lccccc}
\hline & AUC (95\% Cl) & $P$ & ROC cut-off & Sensitivity (\%, 95\% Cl) & Specificity (\%, 95\% Cl) \\
\hline hs-cTnl at presentation & $0.76(0.70-0.81)$ & $<0.001$ & $87.1 \mathrm{ng} / \mathrm{L}$ & $52.8(40.7-64.7)$ & $89.5(84.5-93.3)$ \\
hs-cTnl at 3 hours after presentation & $0.92(0.88-0.95)$ & $<0.001$ & $48.7 \mathrm{ng} / \mathrm{L}$ & $88.9(79.3-95.1)$ & $81.3(75.4-86.4)$ \\
Absolute change & $0.96(0.92-0.98)$ & $<0.001$ & $16.2 \mathrm{ng} / \mathrm{L}$ & $98.6(92.5-99.8)$ & $84.7(79.1-89.3)$ \\
Relative change & $0.89(0.85-0.93)$ & $<0.001$ & $42.1 \%$ & $81.9(71.1-90.0)$ & $80.9(74.9-86.0)$ \\
\hline
\end{tabular}

\section{DISCUSSION}

In this study, we evaluated the diagnostic performance of abso- lute and relative changes in hs-cTnl for the early diagnosis of AMI in patients in the ED. Our study demonstrated that the absolute change at 3 hours after presentation was superior to the 
relative change for diagnosing AMI. The AUC value was 0.96 for the absolute change and 0.89 for the relative change $(P=0.014)$; thus, the absolute change in hs-cTnl was more reliable for diagnosing AMI in patients in the ED. Reichlin, et al. [21] evaluated the diagnostic performance of absolute and relative changes in hs-cTn for AMI diagnosis and demonstrated superiority of the absolute change over the relative change; these findings are consistent with ours.

The hs-cTn assay can detect small amounts of cTn; thus, the initially very low cTn values in patients without AMI were measurable. Moreover, a small change in cTn in patients with initially very low cTn values can readily result in a large relative change, for example, a 50-60\% change. In contrast, some AMI patients might have high baseline cTn values when they visit the ED late after symptom onset. A significant absolute change in cTn might not reach a $20 \%$ relative change in these patients. Thus, a diagnostic method using the relative change in cTn might be affected by the baseline cTn value, which would negatively impact the AMI diagnostic performance. Moreover, detecting cTn at very low values and the use of relative change also dramatically increases the false-positive rate and leads to a potentially large increase in invasive testing and diagnosis of myocardial infarction [22].

The ESC guidelines suggest that changes in the hs-cTn value in the range of $50-60 \%$ when the initial values are $\leq$ the 99th percentile URL or changes of $>20 \%$ when the initial values are $\geq$ the 99th percentile URL be used to diagnose AMI [6, 13]. Several studies that demonstrated the AMI diagnostic performance of absolute changes in hs-cTn are listed in Table 2. These studies, using various hs-cTn assays, demonstrated that the ROCderived absolute changes are useful regardless of the hs-cTn assay used. Interestingly, our study, in line with previous studies, revealed a high sensitivity and indicated good diagnostic performance for ruling out AMI with serial hs-cTn measurements. However, the diagnostic specificity is variable. In addition, cut-off values of the absolute change for defining AMI differ, because these depend on the assay used. Therefore, the ESC could not present a cut-off value for the absolute change in hs-cTn to define AMI [13]. To the best of our knowledge, this is the first study to report an absolute change in hs-cTnl to diagnose AMI in the ED in a Korean population. We report that an absolute change in hs-cTnl >16.2 ng/L at 3 hours after presentation should be considered for AMI diagnosis.

An optimal time interval for the measurement of hs-cTn has not yet been established. ESC guidelines recommend that blood be sampled at presentation and 1-3 hours later, or earlier in the case of hs-cTn assays [13]. The diagnostic performance of hscTn measured with a 2-hour interval has been previously evaluated [21, 23]. Rubini Gimenez, et al. [24] studied rule-in and rule-out of AMI based on 1-hour change in hs-cTn value. Yokoyama, et al. [25] assessed the AMI diagnostic performance of the 30-minute change in hs-cTn value. However, collecting blood for retesting within such a short time may be inconvenient and ineffective in the ED. Further, such a short retest interval may reduce the AMI diagnostic performance [26, 27]. In addition, hs-cTn assays use the 99th percentile as a cut-off, which may result in a low positive predictive value (PPV) for AMI; however, the low PPV could be improved by increasing the retest interval. A few studies have demonstrated a marked improvement in the PPV for AMI of hs-cTn with a 3-hour retest interval [28-30]. For these reasons, the Asia Pacific consensus still recommends measuring hs-cTn at presentation and 3 hours later for the assessment of ACS [31].

This study had some limitations. First, this was a single-center

Table 2. Previous studies on absolute changes in hs-cTnl value for the diagnosis of AMI

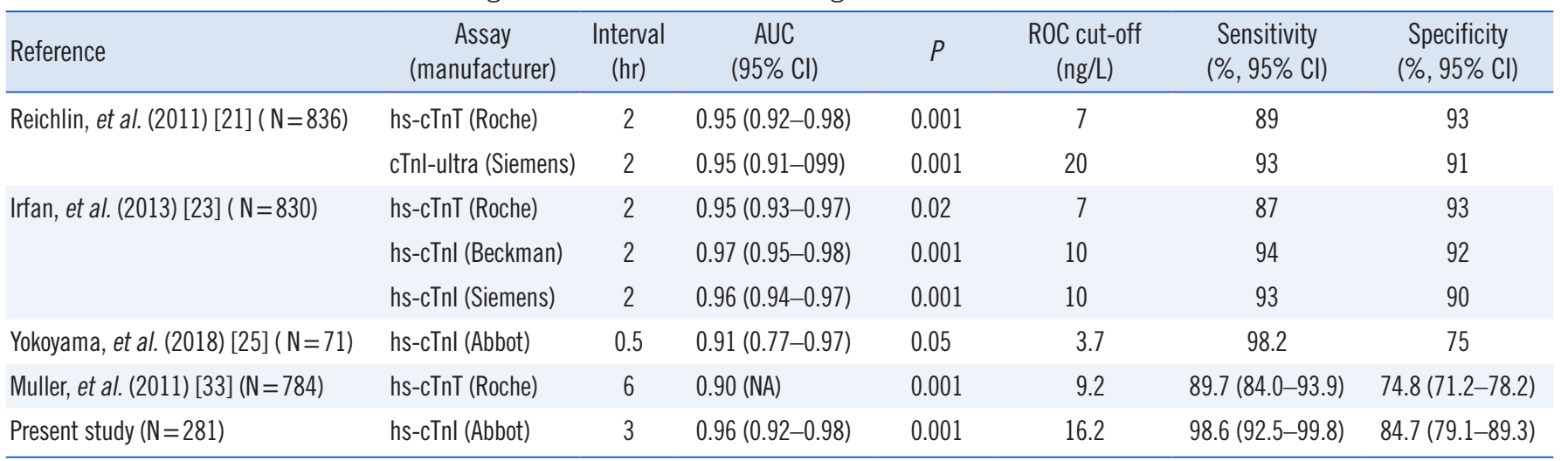

Abbreviations: AMI, acute myocardial infarction; AUC, area under the curve; Cl, confidence interval; hs-cTnl, high-sensitivity cardiac troponin I; hs-cTnT, high-sensitivity cardiac troponin T; ROC, receiver-operating characteristic; NA, not applicable. 
study with a small sample size, and we focused on hs-cTnl; other troponin assays were not evaluated. Second, we assessed AMI using a specific hs-cTnl assay; therefore, our findings cannot be generalized to other facilities and patients. Moreover, when using hs-cTn assays, biological variation needs to be considered [4]. However, we did not evaluate biological variation in hs-cTnl value in the study population. Conjoint analytical and biological variation generally is in the range of $50 \%-60 \%$ [4]. Third, we did not evaluate any clinical benefit associated with the use of the absolute change in hs-cTnl to diagnose AMI in the ED. Further studies using our data are required to validate rule-in and/ or rule-out algorithms for AMI diagnosis. Fourth, the proportion of patients who visited the ED within 3 hours after the onset of chest pain was approximately $40 \%$. However, there was no difference in the delay time among the groups as shown in Table 1. Therefore, this might not have affected our findings. Moreover, while we attempted to collect 2 nd blood samples at 3 hours after presentation at the ED, the median time was closer to 2 hours and a half. Finally, hs-cTn is not a disease-specific biomarker, although it reflects myocardial injury well as a cardiac-specific biomarker [32]. Clinicians should consider individual clinical symptoms and use other diagnostic tools to identify AMI in patients who present with chest pain. Nevertheless, it is essential to check hs-cTn elevation to identify myocardial injury in patients presenting at the ED. The mortality and complication rates were higher in patients with non-ST elevation myocardial infarction than in those with unstable angina, which is myocardial ischemia without myocardial necrosis. In addition, high hs-cTnl value correlated with the risk of major adverse cardiac events including mortality [33]. Therefore, the use of absolute changes in hs-cTn may help clinicians make an early decision on the therapeutic strategy [34].

In conclusion, absolute change in hs-cTnl at 3 hours after presentation in the ED improves the AMI diagnostic performance compared with the relative change. Based on our findings, a rise and/or fall in hs-cTnl of $>16.2 \mathrm{ng} / \mathrm{L}$ seems useful to identify AMI in patients presenting with chest pain at the ED in Korea.

\section{ACKNOWLEDGMENTS}

None.

\section{AUTHOR CONTRIBUTIONS}

KJW performed the experiment, analyzed the data, and wrote the draft; $\mathrm{KH}$ and YYM designed the study and finalized the draft;
LKR and KHJ reviewed the draft and commented on it. All authors have accepted responsibility for the entire content of this manuscript and approved submission.

\section{CONFLICTS OF INTEREST}

The authors have no conflicts of interest to declare.

\section{RESEARCH FUNDING}

This study was supported by the grant and product from Abbott Diagnostics.

\section{ORCID}

Jong Won Kim

Hanah Kim

Yeo-Min Yun

Kyeong Ryong Lee

https://orcid.org/0000-0002-4844-1572

https://orcid.org/0000-0002-3266-638X

https://orcid.org/0000-0002-5485-8331

Hyun Joong Kim

https://orcid.org/0000-0002-6716-2915

https://orcid.org/0000-0003-1636-0193

\section{REFERENCES}

1. Kolansky DM. Acute coronary syndromes: morbidity, mortality, and pharmacoeconomic burden. Am J Manag Care 2009;15:S36-41.

2. Foley AL. Not just chest pain: presenting symptoms of acute coronary syndrome by gender: A research to practice summary. J Emerg Nurs 2019;45:462-4.

3. Katus H, Ziegler A, Ekinci O, Giannitsis E, Stough WG, Achenbach S, et al. Early diagnosis of acute coronary syndrome. Eur Heart J 2017;38: 3049-55.

4. Thygesen K, Alpert JS, Jaffe AS, Chaitman BR, Bax JJ, Morrow DA, et al. Fourth universal definition of myocardial infarction (2018). Circulation 2018;138:e618-51.

5. Apple FS, Jaffe AS, Collinson P, Mockel M, Ordonez-Llanos J, Lindahl B, et al. IFCC educational materials on selected analytical and clinical applications of high sensitivity cardiac troponin assays. Clin Biochem 2015; 48:201-3.

6. Thygesen K, Mair J, Giannitsis E, Mueller C, Lindahl B, Blankenberg S, et al. How to use high-sensitivity cardiac troponins in acute cardiac care. Eur Heart J 2012;33:2252-7.

7. Thygesen K, Mair J, Katus H, Plebani M, Venge P, Collinson P, et al. Recommendations for the use of cardiac troponin measurement in acute cardiac care. Eur Heart J 2010;31:2197-204.

8. Sugiura T, Dohi Y, Takase H, Yamashita S, Mizoguchi T, Fujii S, et al. Close association between circulating high-sensitivity cardiac troponin I and metabolic syndrome in the general population. Hypertens Res 2019; 42:1768-75.

9. Rangaswami J, Bhalla V, Blair JEA, Chang TI, Costa S, Lentine KL, et al. Cardiorenal syndrome: classification, pathophysiology, diagnosis, and treatment strategies: A scientific statement from the American Heart Association. Circulation 2019;139:e840-78.

10. Ooi DS, Isotalo PA, Veinot JP. Correlation of antemortem serum creatine 
kinase, creatine kinase-MB, troponin I, and troponin T with cardiac pathology. Clin Chem 2000;46:338-44.

11. Collinson PO, Apple F, Jaffe AS. Use of troponins in clinical practice: evidence in favour of use of troponins in clinical practice. Heart 2020; 106:253-5.

12. Vasile VC and Jaffe AS. High-sensitivity cardiac troponin for the diagnosis of patients with acute coronary syndromes. Curr Cardiol Rep 2017; 19:92.

13. Roffi M, Patrono C, Collet JP, Mueller C, Valgimigli M, Andreotti F, et al. 2015 ESC guidelines for the management of acute coronary syndromes in patients presenting without persistent ST-segment elevation: task force for the management of acute coronary syndromes in patients presenting without persistent ST-segment elevation of the European Society of Cardiology (ESC). Eur Heart J 2016;37:267-315.

14. Pickering JW, Greenslade JH, Cullen L, Flaws D, Parsonage W, Aldous S, et al. Assessment of the European Society of Cardiology 0-hour/1-hour algorithm to rule-out and rule-in acute myocardial infarction. Circulation 2016;134:1532-41.

15. Jaffe AS, Moeckel M, Giannitsis E, Huber K, Mair J, Mueller C, et al. In search for the holy grail: suggestions for studies to define delta changes to diagnose or exclude acute myocardial infarction: a position paper from the study group on biomarkers of the acute cardiovascular care association. Eur Heart J Acute Cardiovasc Care 2014;3:313-6.

16. Agewall S, Giannitsis E, Jernberg T, Katus H. Troponin elevation in coronary vs. non-coronary disease. Eur Heart J 2011;32:404-11.

17. Kavsak PA, Ko DT, Wang X, Macrae AR, Jaffe AS. Increasing cardiac troponin changes measured by a research high-sensitivity troponin I assay: absolute vs percentage changes and long-term outcomes in a chest pain cohort. Clin Chem 2010;56:1902-4.

18. Apple FS, Pearce LA, Smith SW, Kaczmarek JM, Murakami MM. Role of monitoring changes in sensitive cardiac troponin I assay results for early diagnosis of myocardial infarction and prediction of risk of adverse events. Clin Chem 2009;55:930-7.

19. NACB Writing Group, Wu AH, Jaffe AS, Apple FS, Jesse RL, Francis GL et al. National Academy of Clinical Biochemistry laboratory medicine practice guidelines: use of cardiac troponin and B-type natriuretic peptide or N-terminal proB-type natriuretic peptide for etiologies other than acute coronary syndromes and heart failure. Clin Chem 2007;53:208696.

20. Ji M, Moon HW, Hur M, Yun YM. Determination of high-sensitivity cardiac troponin I 99th percentile upper reference limits in a healthy Korean population. Clin Biochem 2016;49:756-61.

21. Reichlin T, Irfan A, Twerenbold R, Reiter M, Hochholzer W, Burkhalter H, et al. Utility of absolute and relative changes in cardiac troponin concentrations in the early diagnosis of acute myocardial infarction. Circu- lation 2011;124:136-45

22. Fitzgerald G, Kerley RN, Kiernan TJ. High-sensitivity troponin assays: development and utility in a modern health-care system. Expert Rev Cardiovasc Ther 2019;17:763-70.

23. Irfan A, Reichlin T, Twerenbold R, Meister M, Moehring B, Wildi K, et al. Early diagnosis of myocardial infarction using absolute and relative changes in cardiac troponin concentrations. Am J Med 2013;126:781-88.e2.

24. Rubini Gimenez M, Twerenbold R, Jaeger C, Schindler C, Puelacher C, Wildi $\mathrm{K}$, et al. One-hour rule-in and rule-out of acute myocardial infarction using high-sensitivity cardiac troponin I. Am J Med 2015;128:86170.e4.

25. Yokoyama H, Higuma T, Endo T, Nishizaki F, Hanada K, Yokota T, et al. "30-minute-delta" of high-sensitivity troponin I improves diagnostic performance in acute myocardial infarction. J Cardiol 2018;71:144-8.

26. Mueller $\mathrm{C}$, Twerenbold R, Reichlin T. Early diagnosis of myocardial infarction with sensitive cardiac troponin assays. Clin Chem 2019;65:490-1.

27. Reichlin T, Hochholzer W, Bassetti S, Steuer S, Stelzig C, Hartwiger S, et al. Early diagnosis of myocardial infarction with sensitive cardiac troponin assays. N Engl J Med 2009;361:858-67.

28. Gualandro DM, Twerenbold R, Boeddinghaus J, Nestelberger T, Puelacher C, Müller C. Biomarkers in cardiovascular medicine: towards precision medicine. Swiss Med Wkly 2019;149:w20125.

29. Wildi K, Nelles B, Twerenbold R, Rubini Giménez M, Reichlin T, Singeisen $\mathrm{H}$, et al. Safety and efficacy of the $0 \mathrm{~h} / 3 \mathrm{~h}$ protocol for rapid rule out of myocardial infarction. Am Heart J 2016;181:16-25.

30. Keller T, Zeller T, Ojeda F, Tzikas S, Lillpopp L, Sinning C, et al. Serial changes in highly sensitive troponin I assay and early diagnosis of myocardial infarction. JAMA 2011;306:2684-93.

31. Tan JWC, Lam CSP, Kasim SS, Aw TC, Abanilla JM, Chang WT, et al. Asia-pacific consensus statement on the optimal use of high-sensitivity troponin assays in acute coronary syndromes diagnosis: focus on hsTnl. Heart Asia 2017;9:81-7.

32. Garg P, Morris P, Fazlanie AL, Vijayan S, Dancsi B, Dstidar AG, et al. Cardiac biomarkers of acute coronary syndrome: from history to high sensitivity cardiac troponin. Intern Emerg Med 2017;12:147-55.

33. Marino R, Magrini L, Orsini F, Russo V, Cardelli P, Salerno G, et al. Comparison between soluble ST2 and high-sensitivity troponin I in predicting short-term mortality for patients presenting to the emergency department with chest pain. Ann Lab Med 2017;37:137-46.

34. Muller M, Bierner M, Vafaie M, Doerr S, Keller T, Blankenberg S, et al. Absolute and relative kinetic changes of high-sensitivity cardiac troponin $T$ in acute coronary syndrome and in patients with increased troponin in the absence of acute coronary syndrome. Clin Chem 2012;58: 209-18. 